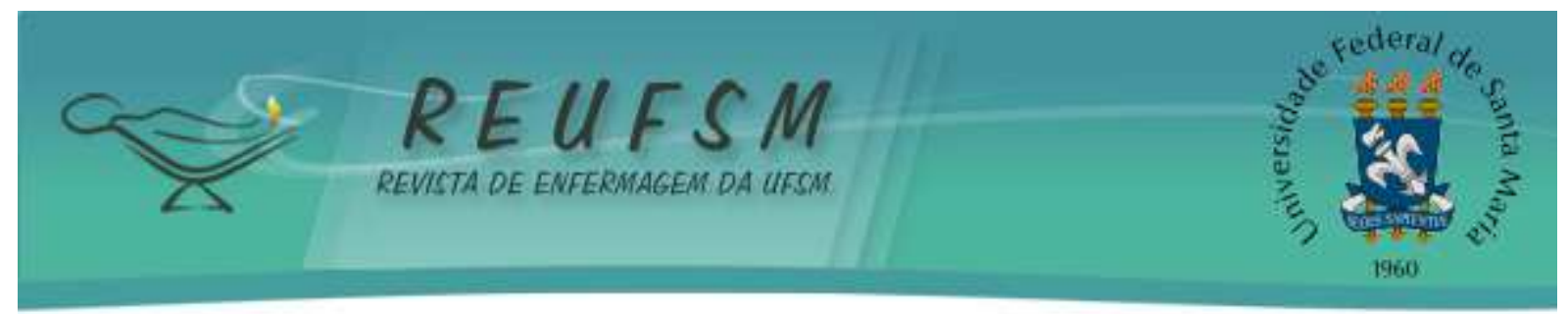

ARTIGO ORIGINAL

\title{
ADMINISTRAÇÃO DE MEDICAMENTOS: ERROS COMETIDOS POR PROFISSIONAIS DE ENFERMAGEM E CONDUTAS ADOTADAS
}

\section{DRUG ADMINISTRATION: ERRORS COMMITTED BY NURSING PROFESSIONALS AND ADOPTED CONDUCTS}

\section{ADMINISTRACIÓN DE MEDICINAS: ERRORES COMETIDOS POR PROFESIONALES DE ENFERMERÍA Y CONDUCTAS ADOPTADAS}

\author{
Marcus Vinicius da Rocha Santos da Silva ${ }^{1}$ \\ Francidalma Soares Sousa Carvalho Filha ${ }^{2}$ \\ Tairo Barros Branco ${ }^{3}$ \\ Jessielly Taís Ferreira Guimarães ${ }^{4}$ \\ Najra Danny Pereira Lima ${ }^{5}$ \\ Anne Karollyne Castro Alves ${ }^{6}$
}

\section{Doi: $10.5902 / 2179769225686$}

RESUMO: Objetivo: analisar condutas que devem ser adotadas por profissionais de enfermagem e gestores para que a ocorrência de erros na administração de medicamentos diminua e identificar erros cometidos por profissionais de enfermagem em relação à administração medicamentosa. Método: estudo quantitativo, exploratório descritivo, realizado com 162 profissionais de enfermagem atuantes em um hospital de um município do Maranhão, Brasil. Os dados foram coletados entre março e maio de 2015, analisados por estatística descritiva e utilizou-se o teste do Qui-quadrado para analisar a associação entre variáveis. Resultados: o treinamento e a capacitação foram considerados por $84 \%$ dos trabalhadores como condutas prioritárias a serem adotadas por parte da gestão para que a ocorrência de erros diminua. Constatou-se que 19,6\% dos profissionais já erraram, sendo que medicamento incorreto e diluição inadequada foram as falhas mais citadas. Conclusão: o conhecimento sobre fatores causais de erros na administração medicamentosa é imprescindível para que sejam elaboradas medidas preventivas.

Descritores: Erros de medicação; Conhecimento; Segurança do paciente; Enfermagem.

ABSTRACT: Aim: to analyze behaviors that must be adopted by nursing professionals and managers so that the occurrence of errors in medication administration decreases and to identify errors made by nursing professionals regarding medication administration. Method: descriptive exploratory quantitative study carried out with 162 nursing professionals who work in a hospital in

\footnotetext{
${ }^{1}$ Enfermeiro. Pós-graduação em Docência do Ensino Superior. Instituto de Ensino Superior Franciscano. Caxias, MA, Brasil. E-mail: marcusvinicius.darocha@yahoo.com.br

${ }^{2}$ Enfermeira. Doutora em Saúde. Universidade Estadual do Maranhão. Faculdade de Ciências e Tecnologia do Maranhão. Caxias, MA, Brasil. E-mail: francidalmafilha@gmail.com

${ }^{3}$ Enfermeiro. Pós-graduação em Docência do Ensino Superior. Instituto de Ensino Superior Franciscano. Caxias, MA, Brasil. E-mail: tairopk@msn.com

4 Enfermeira. Faculdade de Ciências e Tecnologia do Maranhão. Caxias, MA, Brasil. E-mail: jessiellytais@hotmail.com

${ }^{5}$ Enfermeira. Pós-Graduação em Neuropsicopedagogia. Faculdade de Ciências, Educação, Saúde, Pesquisa e Gestão. Caxias, MA, Brasil. E-mail: arjandanny@ hotmail.com

6 Enfermeira. Faculdade de Ciências e Tecnologia do Maranhão. Caxias, MA, Brasil. E-mail: annekastroo@hotmail.com
} 


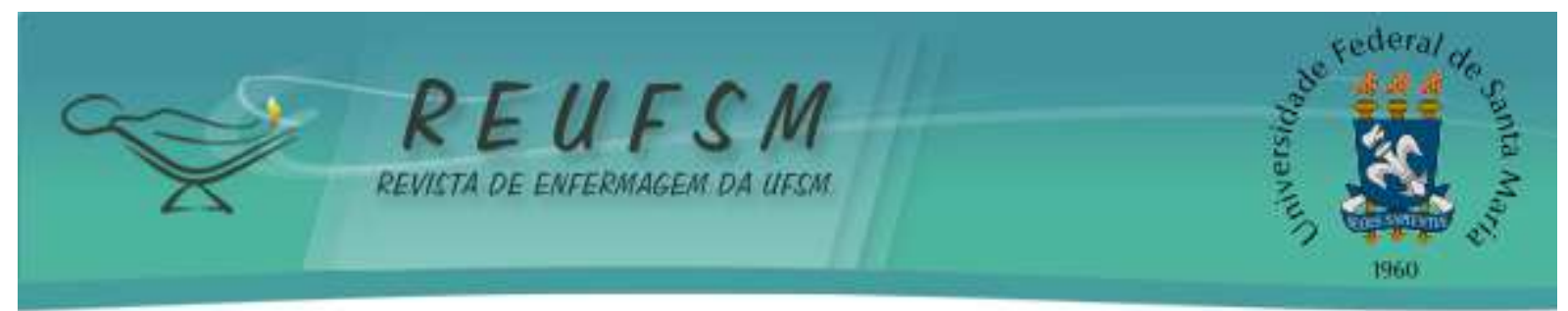

a municipality in Maranhão, Brazil. Data were collected from March to May 2015 and analyzed by descriptive statistics. The Chi-square test was used to analyze the association between variables. Results: training and qualification were considered by $84 \%$ of the workers as a priority conduct to be adopted by the management so that the occurrence of errors decreases. It was verified that $19.6 \%$ of the professionals have already made mistakes, considering that incorrect medication and inadequate dilution were the most cited failures. Conclusion: To know the causing factors for errors in drug administration is essential for preventive measures to be developed.

Descriptors: Medication errors; Knowledge; Patient safety; Nursing.

RESUMEN: Objetivo: analizar conductas que deben ser adoptadas por profesionales de enfermería y gestores para la disminución de la ocurrencia de errores en la administración de medicinas e identificar los errores cometidos por los profesionales de enfermería en relación a la administración de medicinas. Metodo: estudio cuantitativo, exploratorio descriptivo, realizado con 162 profesionales de enfermería actuantes en un hospital de un municipio del Maranhão, Brasil. Los datos fueron recolectados entre marzo y mayo de 2015, analizados por estadística descriptiva y se utilizó la prueba del Qui-cuadrado para analizar la asociación entre variables. Resultados: entrenamiento y capacitación fueron considerados por el $84 \%$ de los trabajadores las medidas prioritarias que deben ser adoptadas por la administración para la disminución de la ocurrencia de errores. Se constató que 19,6\% de los profesionales ya cometieron errores, y la medicación incorrecta e inadecuada dilución fueron los fracasos más citados. Conclusión: el conocimiento sobre factores que causan los errores en la administración de medicinas es imprescindible para que sean elaboradas medidas preventivas. Descriptores: Errores de medicinas; Conocimiento; La seguridad del paciente; Enfermería.

\section{INTRODUÇÃO}

O preparo e a administração de fármacos estão incluídos no rol de atribuições que devem ser implementadas pela equipe de enfermagem, sendo o seu desempenho relevante, por tratar-se de uma responsabilidade da equipe no que concerne aos cuidados prestados aos pacientes. ${ }^{1}$ Salienta-se que erros na administração de medicamentos ocasionam impactos negativos para pacientes e familiares, pois podem provocar incapacidade, prolongar o tempo de internação e recuperação, e em caso de maior gravidade, o cliente pode evoluir para óbito. ${ }^{2}$

É essencial que os profissionais de enfermagem analisem a sua atuação profissional e desenvolvam a assistência na terapêutica medicamentosa baseada em práticas que preservem a segurança dos pacientes, objetivando promover um cuidado satisfatório e qualificado. Menciona-se que a administração de medicamentos é complexa, por essa razão a ocorrência de erros no decorrer do processo pode provocar danos à saúde da pessoa, além de comprometer a equipe multiprofissional e a instituição. ${ }^{3}$

Análise transversal realizada no Canadá, cujo objetivo foi descrever as frequências de intercorrências relacionadas ao cuidado de enfermagem, demonstrou que um em cada sete 


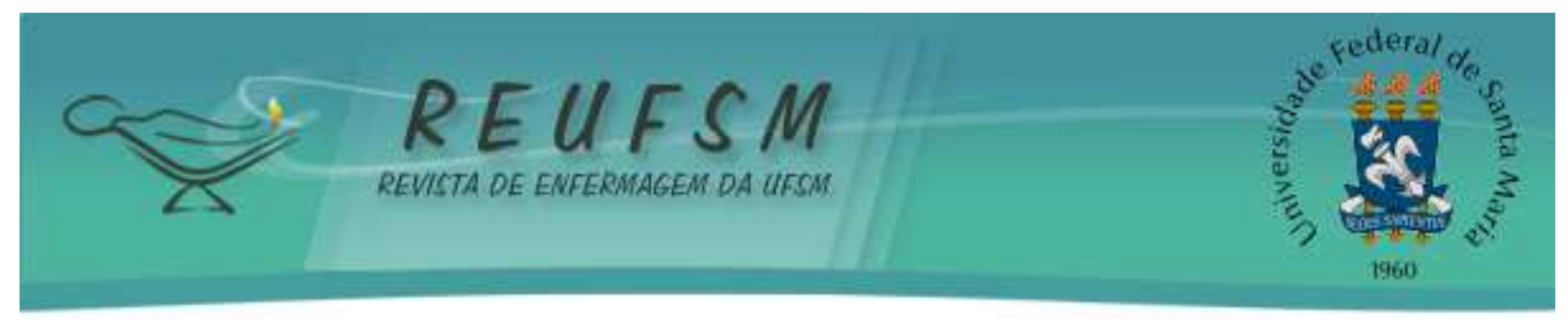

pacientes hospitalizados em unidades de internação clínica foi vítima de, no mínimo, um evento relacionado à assistência prestada, representando uma taxa de incidência de 18,5 por 1000 pacientes/dia. Os achados indicaram que os episódios danosos mais frequentes foram quedas $(5,9 \%)$ e erros na administração de fármacos $(5,5 \%){ }^{4}$

Estudo realizado no Brasil com pacientes internados em um hospital público no município de Brasília identificou que, de um total de 484 doses de medicamentos observadas, verificaram-se erros durante a administração em 69,5\% delas. Tal constatação indica a necessidade de gerenciar o risco de falhas na medicação por meio da interposição de barreiras de segurança entre perigos e possíveis erros. ${ }^{5}$

Reitera-se que a segurança do paciente é aspecto crucial para a garantia da qualidade da assistência à saúde, por isso a adoção de práticas de segurança na terapia medicamentosa representa fator de impacto positivo na redução da incidência de danos e diminuição do tempo de tratamento e hospitalização. Ratifica-se que o desenvolvimento de uma prática de enfermagem consistente, fundamentada no conhecimento científico, simboliza uma importante ferramenta para a consolidação de práticas seguras no que concerne à administração medicamentosa. ${ }^{6}$ A enfermagem deve fornecer cuidado seguro, livre de qualquer dano, detectando no sistema de saúde as possíveis falhas, tornando-se uma busca constante de soluções que visem uma atenção à saúde efetiva. ${ }^{7}$

Os pacientes dirigem-se ao serviço de saúde em busca de tratamento e esperam receber alta em melhores condições do que adentraram, desse modo, a qualidade, a promoção contínua da segurança e a resolutividade da terapêutica necessitam ser asseguradas pela equipe de enfermagem a todos os usuários. Nessa lógica, para que o seguimento de uma assistência sem intercorrências seja obtido, alguns cuidados precisam ser praticados com o intuito de prevenir erros e danos aos pacientes como, por exemplo, o treinamento permanente e a educação continuada da equipe permitindo, assim, a atualização e aprimoramento de habilidades dos profissionais. ${ }^{1}$

Frente ao exposto, a problemática apoiou-se nas seguintes questões: Quais condutas devem ser adotadas por profissionais de enfermagem e gestores para que a ocorrência de erros na administração de medicamentos diminua? Quais erros os profissionais de enfermagem já cometeram no tocante à administração medicamentosa? Neste sentido, esta pesquisa objetivou analisar as condutas que devem ser adotadas por profissionais de enfermagem e gestores para 


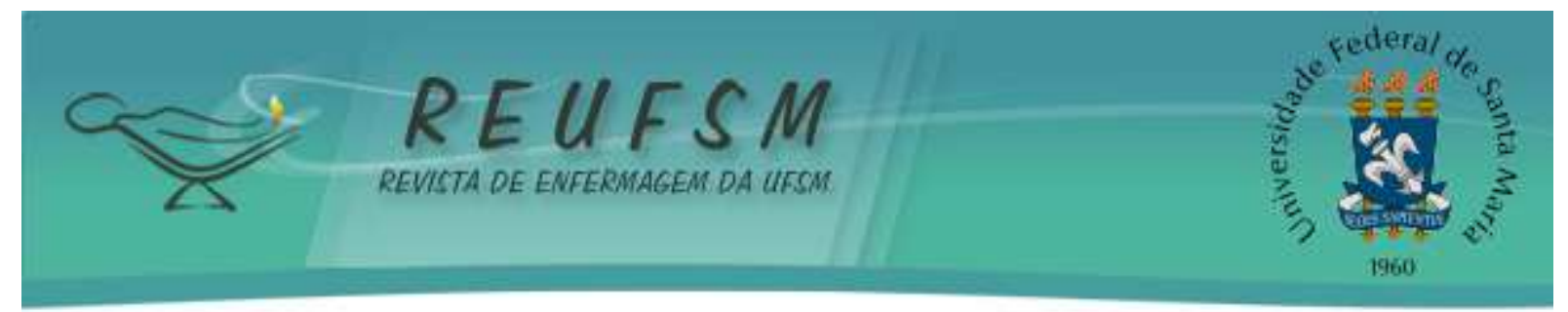

que a ocorrência de erros na administração de medicamentos diminua e identificar erros cometidos pelos profissionais de enfermagem em relação à administração medicamentosa.

\section{MÉTODO}

O presente estudo foi extraído do Relatório Final do Projeto de Pesquisa de Iniciação Científica/ Programa Institucional de Bolsas de Iniciação Científica (PIBIC)/Faculdade de Ciências e Tecnologia do Maranhão (FACEMA), intitulado "Administração de medicamentos: conhecimento, prática e responsabilidades da equipe de enfermagem” vigente de novembro de 2014 a agosto de 2015. Trata-se de um estudo quantitativo, exploratório descritivo.

O cenário da investigação foi o Hospital Geral (HG) de um município do Maranhão (MA), Brasil. O referido hospital foi escolhido por se tratar do maior centro hospitalar do município e contar com uma importante demanda ambulatorial e de internação. Conta com 122 leitos distribuídos entre clínica geral (59), clínica cirúrgica (38), leitos para pacientes com Vírus da Imunodeficiência Humana (HIV)/Síndrome da Imunodeficiência Adquirida (AIDS) (04), unidade de cuidados intermediários (03), unidade de terapia intensiva (09), observação (06) e serviço de pronto atendimento (03).

$\mathrm{Na}$ instituição existem 363 colaboradores: 70 médicos, 01 biomédico, 30 enfermeiros, 07 fisioterapeutas, 06 bioquímicos, 03 assistentes sociais, 02 nutricionistas, 140 técnicos de enfermagem, 44 auxiliares de enfermagem, 02 técnicos vacinadores, 02 auxiliares de nutrição, 07 auxiliares de laboratório, 01 auxiliar administrativo e 48 auxiliares operacionais de serviços diversos.

Os critérios de inclusão dos profissionais de enfermagem participantes deste estudo foram: atuarem profissionalmente no HG há, pelo menos um ano, e possuírem idade igual ou superior a 18 anos. Foram excluídos da investigação os profissionais de enfermagem que durante o período de coleta de dados encontraram-se afastados (licença para tratamento de saúde e férias).

Optou-se por realizar a pesquisa com todos os 214 profissionais de enfermagem (30 enfermeiros, 140 técnicos de enfermagem e 44 auxiliares de enfermagem). Entretanto, 13 foram excluídos [auxiliares de enfermagem - férias (02), auxiliar de enfermagem - licença para tratamento de saúde (01), técnicos de enfermagem - férias (08), técnicos de enfermagem - licença para tratamento de saúde (02)]. 


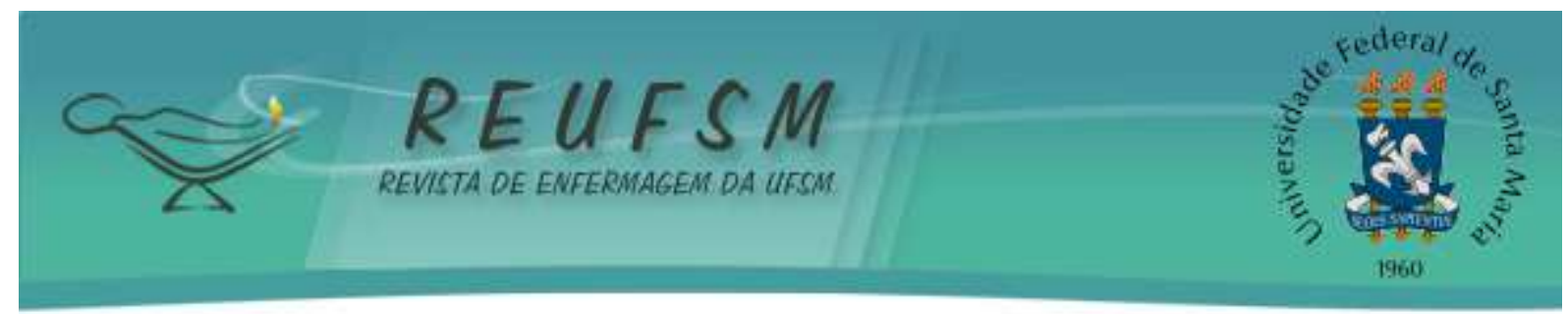

Assim, a população elegível do estudo foi composta por 201 profissionais de enfermagem (30 enfermeiros, 130 técnicos de enfermagem e 41 auxiliares de enfermagem). Destes 201 profissionais, 162 (22 enfermeiros, 102 técnicos de enfermagem e 38 auxiliares de enfermagem) aceitaram participar da pesquisa, enquanto que 08 enfermeiros, 28 técnicos de enfermagem e 3 auxiliares de enfermagem não aceitaram participar do estudo, totalizando 39 perdas.

Ressalta-se que a coleta de dados foi realizada pelos autores da pesquisa e aconteceu no período de março a maio de 2015, durante os plantões no referido serviço de saúde. Enfatiza-se que os participantes da pesquisa receberam orientações referentes aos objetivos da investigação e assinaram o Termo de Consentimento Livre e Esclarecido em duas vias. Ademais, os pesquisadores mantiveram-se à distância dos partícipes durante o procedimento de coleta de dados, para não interferir no processo, nem tampouco intimidá-los. Além disso, quando o profissional não pôde responder aos questionamentos naquele dia e/ou momento, o instrumento de coleta de informações não foi entregue, com vistas a manter a fidelidade das respostas. Por essa razão, retornou-se em oportunidades posteriores, até alcançar todos os participantes do estudo.

Como instrumento de pesquisa, utilizou-se um questionário elaborado pelos investigantes, o qual continha 18 itens/questões fechados (as) acerca das condutas a serem adotadas por profissionais de enfermagem e gestores para que a ocorrência de erros na administração de medicamentos fosse minimizada e também haviam questões que indagavam a respeito do cometimento de falhas na administração de fármacos por parte dos profissionais.

Após a aplicação do questionário, os dados foram organizados em um banco de dados, digitados no software Statistical Package for the Social Sciences - SPSS (versão 20.0 for Windows), posteriormente, consolidados por meio das técnicas de estatísticas descritivas e apresentados em forma de tabelas.

O teste Qui-quadrado foi utilizado para verificar a associação entre a variável dependente (categoria profissional - enfermeiro, técnicos e auxiliares de enfermagem) e as demais variáveis do estudo (condutas que devem ser adotadas para diminuir a ocorrência de erros em relação ao profissional envolvido e pela chefia/gestão, cometimento de erros por profissionais de enfermagem no que tange à administração de medicamentos), sendo adotado o valor de $\mathrm{p}<0,05$ para considerar uma associação estatisticamente significativa. Procedeu-se, em seguida, a análise e discussão dos achados com base na literatura produzida sobre o tema. 


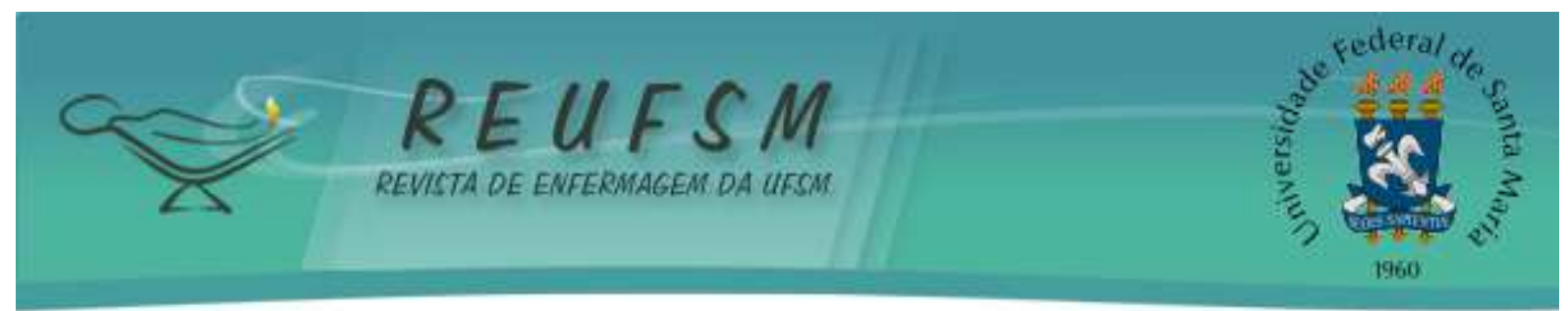

Os aspectos éticos da pesquisa foram amparados pela Resolução do Conselho Nacional de Saúde $n^{\circ} 466$ de 12 de dezembro de 2012. O projeto foi submetido à apreciação de Comitê de Ética de Pesquisa, sendo aprovado em 05 de março de 2015 sob o parecer $n^{\circ}$ 974.945 e Certificado de Apresentação para Apreciação Ética no 39778114.9.0000.5554.

\section{RESULTADOS E DISCUSSÃO}

Participaram do estudo 162 profissionais de enfermagem, dentre os quais 149 (92\%) são do sexo feminino e 13 (8\%) são do sexo masculino. Em relação à faixa etária, constatouse que $66(40,8 \%)$ profissionais situam-se entre 42 a 53 anos e $40(24,7 \%)$ possuem de 36 a 41 anos. Quanto à categoria profissional, detectou-se que $22(13,6 \%)$ são enfermeiros, 102 (63\%) são técnicos de enfermagem e 38 (23,5\%) são auxiliares de enfermagem.

No que concerne à variável que trata do conceito de erro de medicação, percebeu-se que 140 profissionais $(86,4 \%)$ foram corretos em suas colocações ao considerarem a afirmativa correta, enquanto que $22(13,6 \%)$ responderam errado ou não souberam responder (Tabela 1). Entende-se que o fato de a maioria dos profissionais conhecerem acerca do conceito supracitado representa um aspecto positivo, haja vista que tal saber é fundamental para a manutenção da qualidade dos cuidados prestados aos pacientes. ${ }^{8-9}$

Tabela 1: Dados relativos às condutas que devem ser adotadas pelos profissionais de enfermagem e gestores para que haja diminuição da ocorrência de erros na administração de medicamentos segundo a categoria profissional. Caxias (MA), 2015.

\begin{tabular}{|c|c|c|c|c|c|c|c|}
\hline \multirow[t]{2}{*}{ Variáveis } & \multicolumn{2}{|c|}{ Enfermeiro } & \multicolumn{2}{|c|}{$\begin{array}{l}\text { Técnico/ } \\
\text { Auxiliar }\end{array}$} & \multicolumn{2}{|c|}{ Total } & \multirow[t]{2}{*}{$\boldsymbol{P}$} \\
\hline & n & $\%$ & $\mathbf{N}$ & $\%$ & $\mathbf{N}$ & $\%$ & \\
\hline $\begin{array}{l}\text { Erro de medicação é um evento evitável } \\
\text { que pode causar danos ao paciente }\end{array}$ & & & & & & & 0,135 \\
\hline Verdadeiro & 22 & 100,0 & 118 & 84,3 & 140 & 86,4 & \\
\hline Falso & 0 & 0,0 & 09 & 6,4 & 09 & 5,6 & \\
\hline Não sei & 0 & 0,0 & 13 & 9,3 & 13 & 8,0 & \\
\hline $\begin{array}{l}\text { Condutas que devem ser adotadas para } \\
\text { diminuir a ocorrência de erros em relação } \\
\text { ao profissional envolvido e pela } \\
\text { chefia/gestão }\end{array}$ & & & & & & & \\
\hline $\begin{array}{l}\text { Diminuição da sobrecarga de trabalho do } \\
\text { profissional }\end{array}$ & 17 & 77,3 & 75 & 53,6 & 92 & 56,8 & $\mathbf{0 , 0 3 7}$ \\
\hline Profissional mais atento & 20 & 90,9 & 129 & 92,1 & 149 & 92,0 & 0,843 \\
\hline Outras ações dos profissionais & 02 & 9,1 & 14 & 10,0 & 16 & 9,9 & 0,894 \\
\hline Treinamento/capacitação & 22 & 100,0 & 114 & 81,4 & 136 & 84,0 & $\mathbf{0 , 0 2 7}$ \\
\hline Maior supervisão e observação & 16 & 72,7 & 73 & 52,1 & 89 & 54,9 & 0,071 \\
\hline Avaliação do profissional ao admiti-lo & 15 & 68,2 & 35 & 25,0 & 50 & 30,9 & $<0,001$ \\
\hline
\end{tabular}




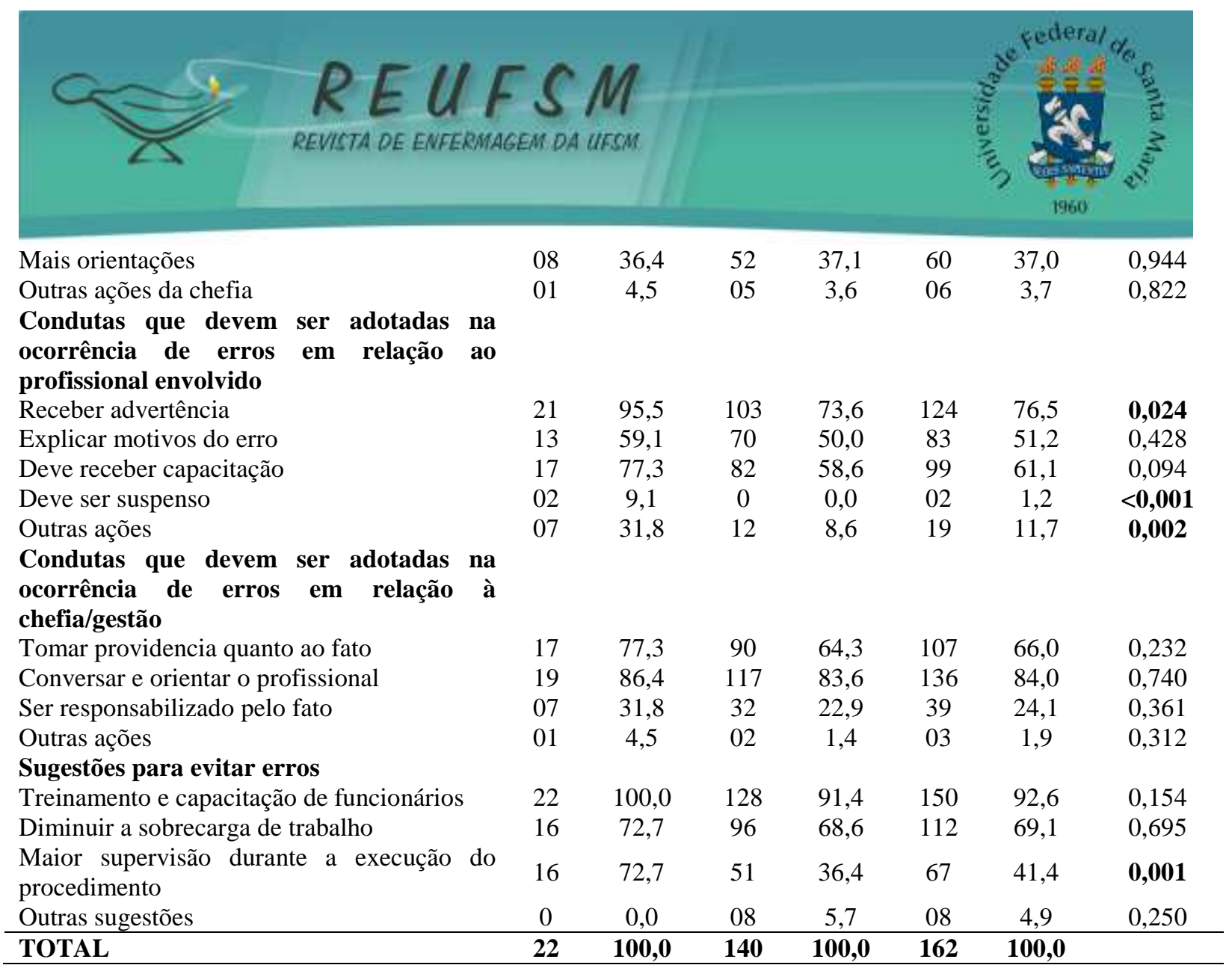

Em se tratando das ações que podem ser desenvolvidas para diminuir a ocorrência de erros na medicação em relação ao profissional detectou-se que 149 (92\%) dos profissionais apontaram a necessidade do profissional estar mais atento, com vistas a diminuir a ocorrência dos erros. No que diz respeito a tais ações envolvendo a chefia/gestão, verificou-se associação estatisticamente significante $(\mathrm{p}=0,027)$ entre a categoria profissional (enfermeiro, técnicos e auxiliares) e o treinamento/capacitação, sendo que $84 \%$ dos profissionais apontaram o treinamento ou a capacitação como fatores essenciais para que diminuam os erros envolvendo a administração de medicamentos.

É válido mencionar que a capacitação da equipe de enfermagem representa uma ação significativa, porque possibilita o aperfeiçoamento das habilidades dos profissionais. Nesse contexto, aponta-se a importância das estratégias interdisciplinares de capacitação enquanto instrumentos embasadores de um processo de medicação seguro e atualizado. ${ }^{6}$

Assim, a capacitação da equipe de enfermagem mostra-se necessária, principalmente quando busca guiar os profissionais para o desenvolvimento, não somente em relação ao aprendizado de habilidades e destrezas, mas, sobretudo, de um aprendizado que gere novas atitudes, soluções, ideias, conceitos e que modifiquem seus hábitos e comportamentos. 


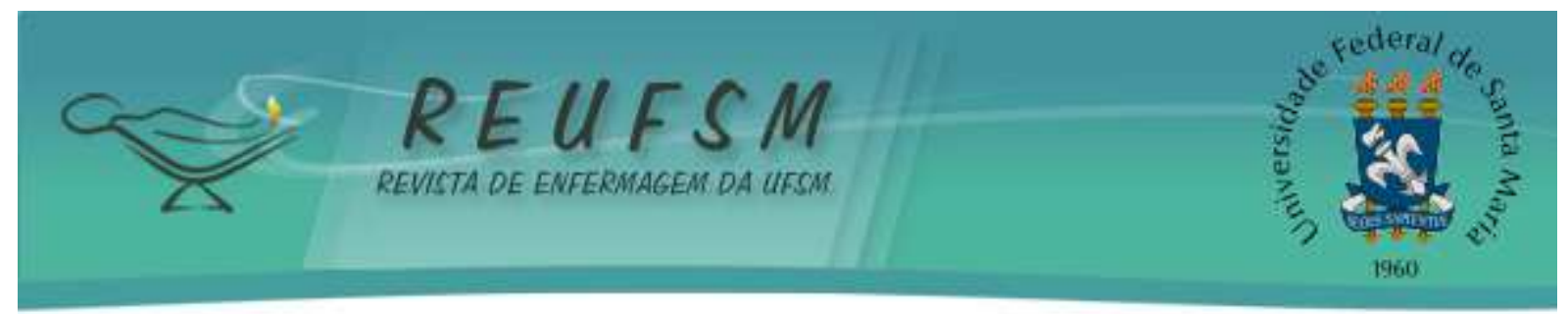

Quanto às condutas que devem ser tomadas na ocorrência de falhas em relação ao profissional envolvido, identificou-se associação estatisticamente significante entre a categoria profissional e o recebimento de advertência $(\mathrm{p}=0,024)$, de modo que a ação supracitada foi apontada por 124 (76,5\%) profissionais. Sobre as atitudes que devem ser adotadas na ocorrência de erros em relação à chefia/gestão, verificou-se que a mais indicada na concepção de $84 \%$ dos profissionais foi que a chefia deve conversar com o profissional e orientá-lo.

Percebe-se que a advertência verbal tem sido a principal ação praticada por instituições diante dos erros na prática da administração de medicamentos. Outrossim, é comum a ausência de atitudes educativas aos profissionais que cometeram esse tipo de falha, expressando que a maioria dos atos administrativos desencadeados na ocorrência de uma falha volta-se para o profissional e não para o desenvolvimento de estratégias que visem aproveitar esse erro e transformá-lo em aprendizado para o sistema. ${ }^{10}$

Quanto às sugestões fornecidas pelos profissionais para prevenir erros de medicação, predominaram o treinamento e a capacitação dos funcionários apontados por $150(92,6 \%)$ profissionais e a diminuição da sobrecarga de trabalho indicada por $112(69,1 \%)$ trabalhadores. Nessa perspectiva, reitera-se a necessidade de educação permanente e capacitação, já que a educação e supervisão contínua representam fatores importantes para uma assistência integral e de qualidade.

A este respeito, evidencia-se o valor da constante atualização e busca pelo conhecimento por parte dos profissionais de saúde, especialmente temas ligados à farmacologia, bem como da implementação de estratégias educativas, tais como discussões em grupo a partir de questionamentos e dúvidas dos profissionais, grupos de estudos, estudos clínicos, reuniões multiprofissionais, cursos de capacitação e elaboração de protocolos para que seja aumentada a segurança na realização do processo da administração de medicamentos. ${ }^{11}$

Quando questionados sobre já terem cometido algum erro na administração de fármacos, verificou-se associação estatisticamente significante entre a categoria profissional e o cometimento de erros na administração de medicamentos $(\mathrm{p}=0,027)$, de maneira que $136(81,4 \%)$ profissionais referiram nunca ter cometido erro de medicação e 26 (19,6\%) profissionais admitiram ter praticado, sendo que os 26 eram técnicos ou auxiliares de enfermagem (Tabela 2).

Esta ocorrência deve-se ao fato de que a administração de fármacos nas instituições hospitalares é atribuição desses profissionais de nível médio que, muitas vezes, realizam essa 


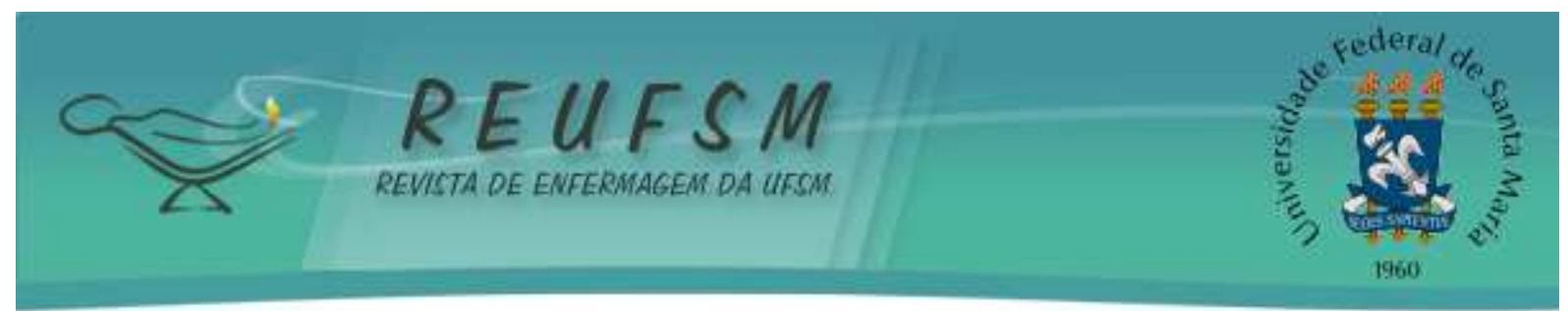

atividade sem supervisão direta do enfermeiro, o qual costuma se envolver com maior frequência em problemas administrativos. Nesse sentido, a falta de supervisão nesse processo por parte dos enfermeiros pode constituir situações de risco para os pacientes. ${ }^{12}$

Tabela 02: Dados relativos ao cometimento de erros por profissionais de enfermagem no que tange à administração de medicamentos segundo a categoria profissional. Caxias-MA, 2015.

\begin{tabular}{|c|c|c|c|c|c|c|c|}
\hline \multirow[t]{2}{*}{ Variáveis } & \multicolumn{2}{|c|}{ Enfermeiro } & \multicolumn{2}{|c|}{$\begin{array}{l}\text { Técnico/ } \\
\text { Auxiliar }\end{array}$} & \multicolumn{2}{|c|}{ Total } & \multirow[t]{2}{*}{$\boldsymbol{P}$} \\
\hline & $\mathbf{n}$ & $\%$ & $n$ & $\%$ & $\mathbf{N}$ & $\%$ & \\
\hline $\begin{array}{l}\text { Já cometeu erro na administração de } \\
\text { medicamento }\end{array}$ & & & & & & & $\mathbf{0 , 0 2 7}$ \\
\hline Sim & 0 & 0,0 & 26 & 19,6 & 26 & 19,6 & \\
\hline Não & 22 & 100,0 & 114 & 81,4 & 136 & 81,4 & \\
\hline TOTAL & 22 & 100,0 & 140 & 100,0 & 162 & 100,0 & \\
\hline
\end{tabular}

Quanto ao tipo de erro cometido, identificou-se que medicamento incorreto foi o erro praticado por $8(30,8 \%)$ profissionais e outros $8(30,8 \%)$ afirmaram já ter falhado na diluição das medicações (Tabela 3). Sobre isso, é relevante mencionar que erros de dosagem, diluição com quantidade inadequada de solução, checagem incorreta, horário equivocado, efeitos tóxicos e irritativos dos medicamentos em uso, prescrição ilegível, interação medicamentosa e medicamentos não administrados constituem falhas detectadas quando se trata da administração medicamentosa. ${ }^{13}$

Tabela 03: Dados relativos aos erros praticados por profissionais de enfermagem na administração de medicamentos segundo a categoria profissional. Caxias-MA, 2015.

\begin{tabular}{|c|c|c|c|c|c|c|}
\hline \multirow[t]{2}{*}{ Variáveis } & \multicolumn{2}{|c|}{ Enfermeiro } & \multicolumn{2}{|c|}{$\begin{array}{l}\text { Técnico/ } \\
\text { Auxiliar }\end{array}$} & \multicolumn{2}{|c|}{ Total } \\
\hline & $\mathbf{n}$ & $\%$ & $\mathbf{n}$ & $\%$ & $\mathbf{N}$ & $\%$ \\
\hline \multicolumn{7}{|l|}{ Tipo de erro cometido } \\
\hline Via errada & 0 & 0,0 & 03 & 11,5 & 03 & 11,5 \\
\hline Dose errada & 0 & 0,0 & 02 & 7,7 & 02 & 7,7 \\
\hline Medicamento incorreto & 0 & 0,0 & 08 & 30,8 & 08 & 30,8 \\
\hline Paciente errado & 0 & 0,0 & 04 & 15,4 & 04 & 15,4 \\
\hline Diluição inadequada & 0 & 0,0 & 08 & 30,8 & 08 & 30,8 \\
\hline Outro & 0 & 0,0 & 01 & 3,8 & 01 & 3,8 \\
\hline \multicolumn{7}{|l|}{ Fator causal de erros } \\
\hline Falta de atenção & 0 & 0,0 & 16 & 61,6 & 16 & 61,6 \\
\hline Falta de conhecimento & 0 & 0,0 & 04 & 15,4 & 04 & 15,4 \\
\hline Estresse & 0 & 0,0 & 02 & 7,7 & 02 & 1,2 \\
\hline Negligência ou imprudência & 0 & 0,0 & 01 & 3,8 & 01 & 0,6 \\
\hline Prescrições ilegíveis & 0 & 0,0 & 03 & 11,5 & 03 & 11,5 \\
\hline
\end{tabular}




$\begin{array}{llllll} & & & & & \\ \end{array}$

Evidenciam-se que falhas de doses e administração de medicamentos em horário errado não consistem, eminentemente, em erros praticados pelos profissionais, ao contrário, podem também representar um reflexo do processamento da cadeia medicamentosa como um todo. ${ }^{14}$

Quanto aos fatores causais de erros, $16(61,6 \%)$ profissionais apontaram a falta de atenção como fator principal para a ocorrência dessas incorreções, 04 (15,4\%) indicaram a falta de conhecimento e $03(11,5 \%)$ mencionaram as prescrições ilegíveis.

Enfatiza-se que as principais desatenções relacionadas à prática de administrar fármacos podem decorrer das seguintes situações: outros membros da equipe de enfermagem quando distraem ou interrompem os colegas no ato da administração de medicamentos, bem como conversas e telefonemas no momento em que o procedimento está sendo realizado. ${ }^{15}$

Ademais, outras causas que contribuem para a ocorrência desses eventos danosos incluem: fatores inerentes ao gerenciamento do serviço e da assistência de enfermagem, como o déficit de pessoal, sobrecarga de trabalho, problemas de relacionamento entre a equipe multiprofissional, falta de liderança e de supervisão de enfermagem adequadas, entre outros. ${ }^{16}$ Estudiosos acrescentam que o conhecimento inadequado da medicação, no que tange à terapêutica das drogas, formas alternativas e cálculos de dosagens inadequados também são responsáveis por muitos dos episódios envolvendo falhas na medicação. ${ }^{17}$

No que tange a detecção do erro, 13 (50\%) profissionais relataram ter percebido o erro por conta própria. Esse achado diverge do resultado obtido em outra averiguação, na qual a maior parte dos profissionais pesquisados referiu que o erro foi identificado por um colega de plantão. ${ }^{18}$ 


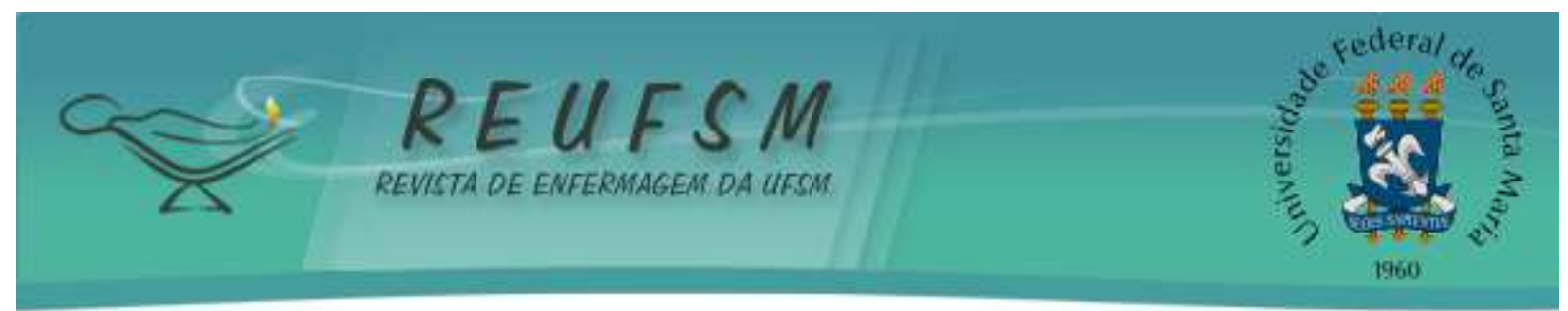

Em se tratando da forma como o paciente evoluiu após a ocorrência desses erros, identificou-se que $24(92,3 \%)$ profissionais referiram que os pacientes envolvidos se recuperaram por completo. Portanto, na presente pesquisa não ocorreram relatos de erros que necessitaram de controle, tratamento, dano permanente ou óbito.

A este respeito, verifica-se que 55,7\% dos eventos relacionados aos erros na medicação resultam em dano temporário aos pacientes, com uma recuperação completa esperada dentro de 12 meses, enquanto 19,1\% causam prejuízo em longo prazo ou resultam em cuidados institucionais ou de enfermagem permanentes. ${ }^{19}$

A partir dessas constatações, enfatiza-se que se houver lesão física ou outro dano à saúde, decorrente, por exemplo, de erros na administração de medicamentos, o profissional terá de indenizar o paciente das despesas do tratamento e dos lucros cessantes até o fim da convalescença. Além disso, se da lesão resultar defeito pelo qual o ofendido não possa exercer seu ofício ou profissão, ou se lhe diminua a capacidade de trabalho, a indenização, além dos itens supracitados, incluirá pensão correspondente à importância do trabalho do qual se inabilitou ou da depreciação que ele sofreu. ${ }^{20}$

Quanto à classe do medicamento envolvido, notou-se a prevalência dos analgésicos e antibióticos, sendo que os mesmos foram indicados respectivamente por $14(53,8 \%)$ e $4(15,4 \%)$ profissionais. Acerca dessa temática, relata-se que os antibióticos, os redutores de acidez gástrica, os anti-hipertensivos e os antitrombóticos apresentam-se como os principais fármacos envolvidos na ocorrência de incorreções relacionadas à implementação da terapia medicamentosa. ${ }^{19,21}$

Destarte, os gestores e as instituições hospitalares devem investir na capacitação dos recursos humanos, ${ }^{22}$ especificamente no que tange a administração de medicamentos, com o intuito de proporcionar condições que garantam a efetiva contribuição dos profissionais de saúde. Dessa forma, será possível prestar uma assistência integral aos pacientes com menor ocorrência de falhas.

\section{CONSIDERAÇÕES FINAIS}

A partir desta pesquisa, verificou-se que, na concepção de $92 \%$ dos profissionais de enfermagem, adotar a conduta de manter-se mais atento durante o ato de administrar medicamentos representa a ação mais relevante para que a ocorrência de erros seja menos frequente. $\mathrm{O}$ treinamento e a capacitação foram considerados por $84 \%$ dos trabalhadores 


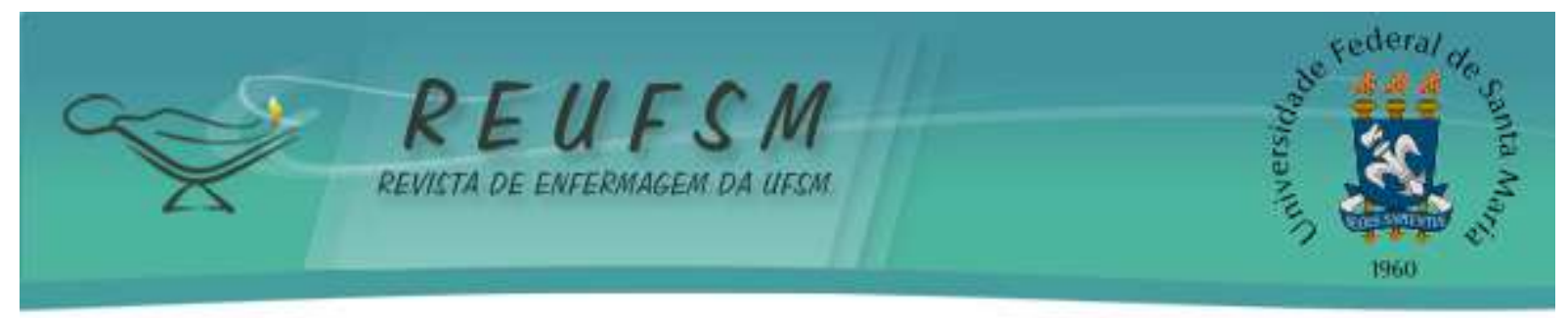

como as condutas prioritárias a serem praticadas por parte da gestão para que falhas de medicação sejam evitadas.

Os resultados obtidos demonstraram que $19,6 \%$ dos profissionais já erraram no tocante à administração de fármacos, sendo que medicamento incorreto e diluição inadequada foram as incorreções mais citadas. Quanto aos fatores causais de erros, 61,6\% dos profissionais apontaram a falta de atenção como fator principal para a ocorrência das falhas.

Após a análise dos resultados deste estudo, observa-se que a administração medicamentosa é uma importante atividade desempenhada pela equipe de enfermagem e o conhecimento sobre os tipos de erros e os fatores causais é significante para que se possa realizar a elaboração de medidas preventivas. É essencial que os erros sejam interpretados como consequências e não como causas.

Esta pesquisa aumenta o entendimento sobre o processo de preparo de medicações pela enfermagem e oferta elementos para colaborar com o cuidado em saúde, objetivando aprimorar a qualidade da assistência prestada ao paciente, suscitando a utilização segura e racional de medicamentos e amparando práticas que evidenciem cada vez menos erros.

Ademais, contribui na produção de conhecimento acerca da segurança do paciente que tem sido bastante debatida no contexto mundial. Diante da análise das incorreções identificadas, recomenda-se que as organizações de saúde trabalhem em busca da cultura de segurança, a qual representa um ponto de partida para que se possa iniciar o planejamento de ações. Para tanto, faz-se necessário o envolvimento da gestão e de todos os atores dos diversos níveis operacionais.

O estudo apresenta como limitação o fato de ter sido realizado em apenas uma instituição, o que não garante a possibilidade de generalização dos resultados. Nesse sentido, recomenda-se a realização de outras investigações acerca da temática, de diferentes métodos, em diversas instituições, para que se possam criar novas estratégias que contribuam para a minimização da ocorrência de incidentes e eventos danosos à saúde dos usuários.

\section{REFERÊNCIAS}

1. Galiza DDF, Moura OF, Barros VL, Luz GOA. Preparo e administração de medicamentos: erros cometidos pela equipe de enfermagem. Rev Bras Farm Hosp Serv Saúde. 2014;5(2):45-50. 


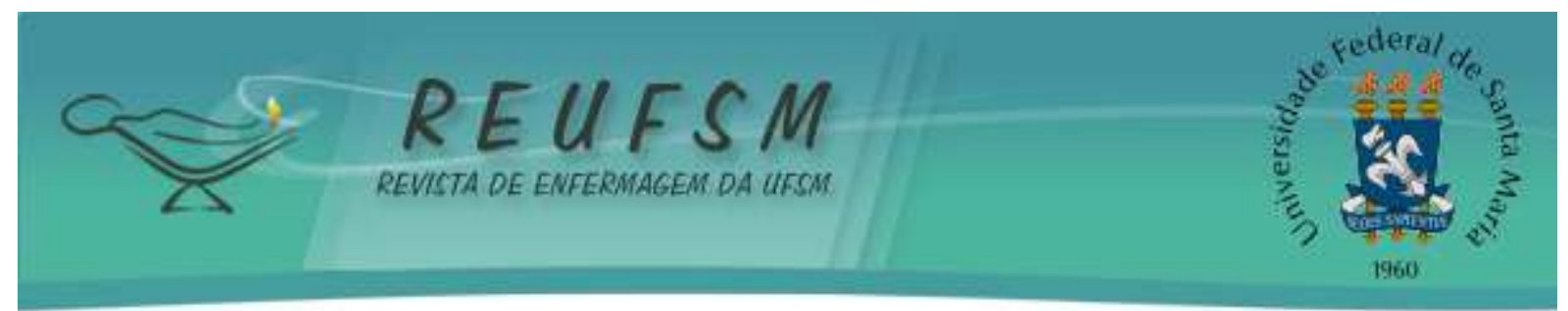

2. Santos DS, Souza OV, Nascimento ALS, Pereira JS, Santos MJC, Alves MC, et al. Segurança do paciente: fatores causais de eventos adversos a medicamentos pela equipe de enfermagem. Cienc Biol Saude. 2014;2(2):19-30.

3. Pereira CDFD, Tourinho FSV, Santos VEP. Segurança do paciente: avaliação do sistema de medicação por enfermeiros utilizando análise fotográfica. Enferm Foco [Internet]. 2016 [acesso em 2017 fev 13];7(1):76-80. Disponível em: http://revista.portalcofen.gov.br/index.php/enfermagem/article/view/672.

4. D'Amour D, Dubois C, Tchouaket E, Clarke S, Blais R. The occurrence of adverse events potentially attributable to nursing care in medical units: cross sectional record review. Int $\mathbf{J}$ Nurs Stud [Internet]. 2014 [acesso em 2017 fev 13];51(6):882-91. Disponivel em: https://www.ncbi.nlm.nih.gov/pubmed/24238893.

5. Volpe CRG, Pinho DLM, Stival MM, Karnikowski MGO. Medication errors in a public hospital in Brazil. Br J Nurs [Internet]. 2014 [acesso em 2017 fev 13];23(11):552-9. Disponivel em: https://www.ncbi.nlm.nih.gov/pubmed/24933543.

6. Praxedes MFS, Telles Filho PCP, Miasso AI, Pereira Júnior AC. Administração de medicamentos: identificação e análise das necessidades educacionais de enfermeiros. Rev Enferm UFPE [Internet]. 2015 [acesso em 2017 fev 13];9(1):76-83. Disponível em: http://www.revista.ufpe.br/revistaenfermagem/index.php/revista/article/view/7319.

7. Dias JD, Mekaro KS, Tibes CMS, Zem-Mascarenhas SH. Compreensão de enfermeiros sobre segurança do paciente e erros de medicação. REME Rev Min Enferm [Internet]. 2014 [acesso em 2016 out 13];18(4):866-73. Disponível em: http://www.reme.org.br/artigo/detalhes/969.

8. Reis GS, Costa JM. Erros de medicação no cotidiano dos profissionais de um hospital de ensino: estudo descritivo exploratório. Rev Bras Farm Hosp Serv Saúde [Internet]. 2012 [acesso em 2017 fev 14];3(2):30-3. Disponível em: http://www.sbrafh.org.br/rbfhss/public/artigos/2012030206BR.pdf.

9. Corbellini VL, Schilling MCL, Frantz SF, Godinho TG, Urbanetto JS. Eventos adversos relacionados a medicamentos: percepção de técnicos e auxiliares de enfermagem. Rev Bras Enferm [Internet]. 2011 [acesso em 2016 nov 27];64(2):241-7. Disponível em: http://www.scielo.br/scielo.php?script=sci_arttext\&pid=S0034-71672011000200004.

10. Praxedes MFS, Telles Filho PCP. Erros e ações praticadas pela instituição hospitalar no preparo e administração de medicamentos. Rev Min Enferm [Internet]. 2011 [acesso em 2016 dez 12];15(3):406-11. Disponível em: http://www.reme.org.br/artigo/detalhes/52.

11. Ferreira MMM, Alves FS, Jacobina FMB. O profissional de enfermagem e a administração segura de medicamentos. Rev Enferm Contemp [Internet]. 2014 [acesso em 2018 mar 15];3(1):619. Disponível em: https://www5.bahiana.edu.br/index.php/enfermagem/article/download/208/300.

12. Teixeira TCA, Cassiani SHB. Análise de causa raiz: avaliação de erros de medicação em um hospital universitário. Rev Esc Enferm USP [Internet]. 2010 mar [acesso em 2016 dez 17];44(1):139-46. Disponível em: http://www.scielo.br/pdf/reeusp/v44n1/a20v44n1.pdf. 


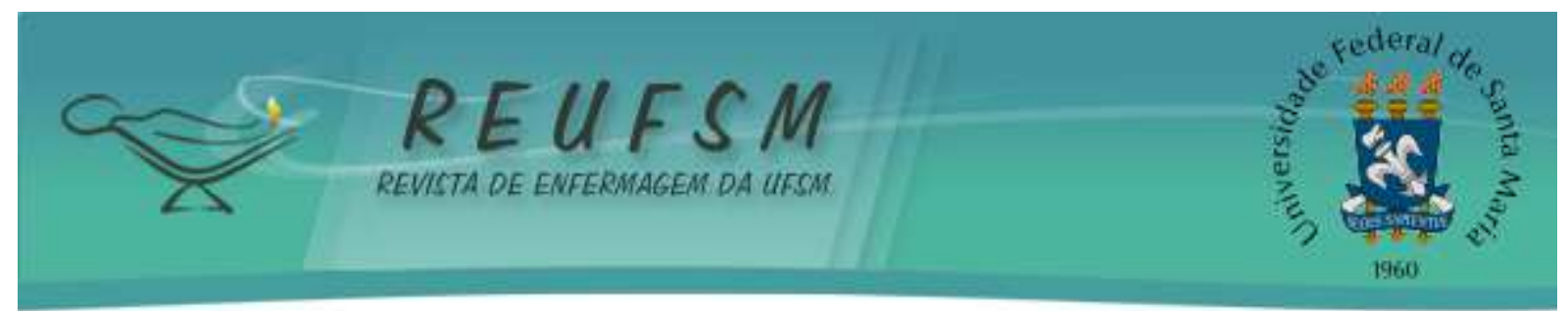

13. Ramos EM. Complicações iatrogênicas no atendimento de emergência de um hospital público. Rev Enferm UFPE [Internet]. 2013 jun [acesso em 2017 jan 03];7(6):4514-20. Disponível em: http://www.revista.ufpe.br/revistaenfermagem/index.php/revista/article/view/4032.

14. Lorenzini E, Santi JAR, Báo ACP. Segurança do paciente: análise dos incidentes notificados em um hospital do sul do Brasil. Rev Gaúcha Enferm [Internet]. 2014 jun [acesso em 2017 jan 03];35(2):121-7. Disponível em: http://www.scielo.br/pdf/rgenf/v35n2/pt_19831447-rgenf-35-02-00121.pdf.

15. Ramos DY, Lesmes VIS. Identificar distrações no processo de administração de medicamentos garante uma prática segura. Av enferm [Internet]. 2014 [acesso em 2017 jan 04];32(1):44-52. Disponível em: http://www.scielo.org.co/pdf/aven/v32n1/v32n1a05.pdf.

16. Duarte SCM, Stipp MAC, Silva MM, Oliveira FT. Eventos adversos e segurança na assistência de enfermagem. Rev Bras Enferm [Internet]. 2015 [acesso em 2017 jan 07];68(1):14454. Disponível em: http://www.scielo.br/pdf/reben/v68n1/0034-7167-reben-68-01-0144.pdf.

17. Mansouri A, Ahmadvand A, Hadjibabaie M, Javadi M, Khoee SH, Dastan F, et al. A review of medication errors in iran: sources, under report in greasons and preventive measures. Iran J Pharm Res [Internet]. 2014 [acesso em 2017 jan 10];13(1):3-17. Disponível: http://ijpr.sbmu.ac.ir/article_1443_68.html.

18. Franco JN, Ribeiro G, D’Innocenzo M, Barros BPA. Percepção da equipe de enfermagem sobre fatores causais de erros na administração de medicamentos. Rev Bras Enferm [Internet]. 2010 [acesso em 2016 dez 22];63(6):927-32. Disponível em: http://www.scielo.br/pdf/reben/v63n6/09.pdf.

19. Marquet K, Claes N, De Troy E, Kox G, Droogmans M, Schrooten W, et al. One fourth of unplanned transfers to a higher level of care are associated with a highly preventable adverse event: a patient record review in six belgian hospitals. Crit Care Med [Internet]. 2015 maio [acesso em 2017 jan 19];43(5):1053-61. Disponível em: https://www.ncbi.nlm.nih.gov/pubmed/25756416.

20. Diniz MH. Curso de direito civil brasileiro: responsabilidade civil. $28^{a}$ ed. São Paulo: Saraiva; 2014. Vol. 7.

21. Bohomol E. Erros de medicação: estudo descritivo das classes dos medicamentos e medicamentos de alta vigilância. Esc Anna Nery [Internet]. 2014 abr-jun [acesso em 2017 jan 21];18(2):311-6. Disponível em: http://www.scielo.br/pdf/ean/v18n2/1414-8145-ean-18-02-0311.pdf.

22. Oliveira WT, Haddad MCL, Vannuchi MTO, Rodrigues AVD, Pissinati PSC. Capacitação de enfermeiros de um hospital universitário público na gestão de custo. Rev Enferm UFSM [Internet]. 2014 jul-set [acesso em 2017 jan 23];4(3):566-74. Disponível em: https://periodicos.ufsm.br/reufsm/article/view/12938/pdf.

Data de submissão: 01/02/2017

Data de aceite: 19/03/2018

Autor correspondente: Marcus Vinicius da Rocha Santos da Silva

Email: marcusvinicius.darocha@yahoo.com.br

Endereço: Rua Sete de Setembro, número 879, Trizidela. Caxias-MA.

CEP: 65607-350 